\title{
Path Planning Based on an Improved Ant Colony Algorithm
}

\author{
Miaomiao Wang ${ }^{1}$, Zhenglin $\mathrm{Li}^{1}$,a , Qing Zhao ${ }^{1}$, Fuyuan $\mathrm{Si}^{1}$ and Dianfang Huang ${ }^{1}$ \\ ${ }^{1}$ College of Electrical and Information Engineering, Guangxi University of Science and Technology, Liuzhou, 545006, China
}

\begin{abstract}
The classical ant colony algorithm has the disadvantages of initial search blindness, slow convergence speed and easy to fall into local optimum when applied to mobile robot path planning. This paper presents an improved ant colony algorithm in order to solve these disadvantages. First, the algorithm use A* search algorithm for initial search to generate uneven initial pheromone distribution to solve the initial search blindness problem. At the same time, the algorithm also limits the pheromone concentration to avoid local optimum. Then, the algorithm optimizes the transfer probability and adopts the pheromone update rule of "incentive and suppression strategy" to accelerate the convergence speed. Finally, the algorithm builds an adaptive model of pheromone coefficient to make the pheromone coefficient adjustment self-adaptive to avoid falling into a local minimum. The results proved that the proposed algorithm is practical and effective.
\end{abstract}

\section{Introduction}

As one of the most important branches of mobile robot, path planning is an important guarantee for mobile robots to successfully complete tasks. The path planning of a mobile robot usually means that a mobile robot can find an optimal path or near optimal path ${ }^{[1]}$ from the starting point to avoid the obstacle arriving at the target point from the starting point in the obstacle environment. At present, the main approaches to solve the path planning problems include artificial potential field ${ }^{[2]}$, immune algorithm [3], genetic algorithm [4], artificial neural network ${ }^{[5]}$ and so on. But these algorithms are complex, locally optimal and inefficient. So it is still of great significance to find a better algorithm or improve the present algorithm.

Ant colony algorithm (ACO) was proposed by $\mathrm{M}$. dorigod in $1991^{[6]}$, it is widely used as an intelligent optimization algorithm to solve the problem of path planning of mobile robot. Ant colony algorithm has the advantages of high efficiency, strong robustness and easy to combine with other algorithms, but it still has the defect ${ }^{[7]}$ which is easy to fall into local optimal solution and slow convergence speed. Therefore, many domestic and foreign scholars began to study the improvement of ant colony algorithm. Hsu successfully solved the problem of ant colony algorithm falling into local optimum by adjusting parameters and updating pheromone continuously ${ }^{[8]}$. But it not solve the initial search blindness problem. $\mathrm{Qu}$ set the pheromone boundary in the algorithm and made some improvements to solves the deadlock problem ${ }^{[9]}$. But the convergence speed of algorithm may be slowly.

In this paper, an improved ant colony algorithm is proposed, which can accelerate the convergence speed by generating uneven initial pheromone distribution and optimizing probability, and avoid local optimum by building an adaptive model of pheromone coefficient.

\section{Robot Environment Map}

The grid method is applied to establish the environment model of mobile robot path planning in this paper. As shown in Figure 1, each cell is divided into two situations based on the coverage of the obstacles: free or occupied. The size of the grid is generally set as the step length of the mobile robot. Each grid is defined as the color property, in which the white represents the passable area and the black represents the obstacle ${ }^{[10]}$.

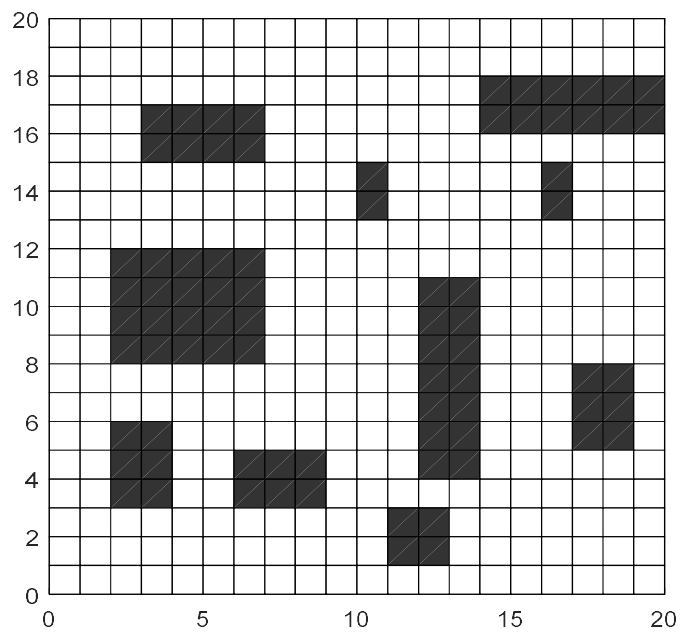

Figure 1. Raster map.

\footnotetext{
a Corresponding author: 1545767299@qq.com
} 


\section{The proposed algorithm}

\subsection{Initialization pheromone}

$A^{*}$ search algorithm ${ }^{[11]}$ is a heuristic algorithm which is widely used to solve the shortest path of point to point in static environment. In this paper, we first define the initial distribution concentration of the global pheromone on the map, and then use the $A^{*}$ algorithm to search the path to increase the pheromone concentration on the path, and the specific initial pheromone setting formula is as follows:

$$
\tau=\left\{\begin{array}{ccc}
\tau_{S}, & \text { if } & L \neq L_{\mathrm{A}} \\
\tau_{S}+\tau_{A} & , \text { if } & L=L_{\mathrm{A}}
\end{array}\right.
$$

$\tau_{\mathrm{s}}$ is the initial distribution concentration of the global pheromone of the ant colony algorithm. The $\tau_{\mathrm{A}}$ is a constant, and it is the result of the $\mathrm{A}^{*}$ algorithm. $\mathrm{L}$ is the walking path of the ant and the $\mathrm{L}_{\mathrm{A}}$ is the path searched by $\mathrm{A}^{*}$ algorithm.

In order to avoid the "precocious" phenomenon caused by the excessive difference between the optimal path and other path pheromones, we restrict the upper and lower bounds of the pheromone concentration on each path according to the MMAS algorithm ${ }^{[12]}$ : each path is set to the maximum value $\tau_{\max }$ and the minimum value $\tau_{\min }$, and the pheromone value must be $\left[\tau_{\min }, \tau_{\max }\right]$. When the current pheromone value is less than the minimum value, $\tau=\tau \mathrm{min}$; When it is greater than the maximum, it is $\tau=\tau \max$. The specific formulas are as follows:

$$
\tau=\left\{\begin{array}{cc}
\tau & \tau_{\min }<\tau<\tau_{\max } \\
\tau_{\min } & \tau \leq \tau_{\min } \\
\tau_{\max } & \tau \geq \tau_{\max }
\end{array}\right.
$$

$\tau_{\max }$ is the upper bound of the pheromone concentration; $\tau_{\min }$ is the lower bound of the pheromone concentration.

\subsection{Optimal transfer probability}

Ant $\mathrm{k}$ which located on the grid $\mathrm{i}$, determines the selection of the next step according to the pheromone on the various paths in the grid environment and the selects heuristic information of the grid $i$ to grid $j$. The corresponding transfer probability is shown as follows:

$$
\begin{aligned}
& p_{i j}{ }^{k}(t)= \\
& \left\{\begin{array}{c}
{\left[\tau_{i j}(t)\right]^{\alpha} \bullet\left[\eta_{i j}(t)\right]^{\beta}} \\
\sum_{\text {seallowed }_{k}}\left[\tau_{i s}(t)\right]^{\alpha} \bullet\left[\eta_{i s}(t)\right]^{\beta} \\
s \in \text { allowed }_{k} \\
0, \text { Others }
\end{array}\right.
\end{aligned}
$$

$\mathrm{s} \in$ allowedk said the next location which the ant $\mathrm{k}$ allows to select. The $\alpha$ is the pheromone enhancement coefficient and the $\beta$ represents the expected heuristic information coefficient. The $\tau_{\mathrm{j}} \mathrm{j}(\mathrm{t})$ is the concentration of the pheromone in the $t$ moment from the current grid $i$ to the lower grid and the $\eta_{\mathrm{ij}}(\mathrm{t})$ is the inspiration information from the current grid to the lower grid $\mathrm{j}$.

It can be seen from the above formula that the heuristic information $\eta_{\mathrm{ij}}=1 / \mathrm{d}_{\mathrm{ij}}$ is the reciprocal of the distance between the two grids, but in the established grid map, the grid is adjacent to each other, and the length gap between different paths is very small, so the heuristic information can not play a certain inspiration. So here, we refer to the heuristic information of the $A^{*}$ algorithm for the distance between the grid and the end of the end as a heuristic information, the reciprocal of the Euclidean distance between the grid $\mathrm{j}$ and the end point:

$$
\eta_{\mathrm{ij}}(t)=\frac{1}{d_{j \mathrm{G}}}
$$

$\mathrm{G}$ represents the target grid node.

In addition, in order to increase the diversity of the solution, the transfer probability formula is improved. The improved algorithm introduces a constant $\mathrm{q} 0$, and the pseudo random proportional rule is used for state transfer. Under this rule, a random number in the $[0,1]$ range is generated whenever the ant is to choose the next node, according to the size of the random number. Determine which way to generate the direction of ant transfer.

$$
\begin{aligned}
& p_{i j}^{\mathrm{k}^{*}}(t)= \\
& \left\{\begin{array}{cc}
\left.\underset{\text { seallowed }}{\arg \max }\left[\tau_{i \text { is }}(t)\right]^{\alpha} \cdot\left[\eta_{i s}(t)\right]^{\beta}\right\} & q \leq q 0 \\
p_{i j}{ }^{k}(t) & q>q 0
\end{array}\right.
\end{aligned}
$$

$\mathrm{q}$ is a uniformly distributed random number in $(0,1)$ and $\mathrm{q} 0$ is a constant of $[0,1]$.

\subsection{Optimization pheromone}

In the classical ACO, $\rho$ is a constant, represents the degree of evaporation on all paths are the same. If the value of $\rho$ is too large, it is easy to fall into local convergence, miss a better solution and affect the global search capability. If the value of $\rho$ is too small, the pheromone on each path will gradually evaporate, which requires more time to search and reduce the speed of convergence.

In this paper, a formula of pheromone volatilization coefficient is proposed. In the later stage of the search, when the ant colony algorithm falls into local convergence, the search space is expanded by reducing the value of $\rho$, and the local convergence is avoided, and the change of the value of the value is as follows:

$$
\begin{aligned}
& \rho(t)= \\
& \left\{\begin{array}{cc}
\left(e^{d(-1)}-1\right) / 2+0.04(1-\rho(t-1))^{2}-0.5 & , \rho>\rho_{\text {min }} \\
\rho_{\text {min }} & \rho \leq \rho_{\text {min }}
\end{array}\right.
\end{aligned}
$$

$\rho$ is the pheromone evaporation coefficient, $\rho_{\min }$ is the minimum pheromone evaporation coefficient. 


\subsection{Update rules of pheromone}

As time goes on, the pheromone trajectory will gradually evaporate, when the ants move from the grid i to the next grid $\mathrm{j}$, the pheromone locally updates $\Delta \tau_{\mathrm{ij}}{ }^{\mathrm{k}}(\mathrm{t})$. When all ants arrive at the target grid $G$ to complete a cyclic movement, the size of the global pheromone value will be updated, the pheromone updating formula is as follows:

$$
\begin{gathered}
\tau_{i j}(t+1)=(1-\rho) \tau_{i j}(t)+\Delta \tau_{i j}{ }^{\mathrm{k}}(t) \\
\Delta \tau_{i j}{ }^{k}(t)=\left\{\begin{array}{c}
\frac{Q}{L_{\mathrm{k}}(t)}, i f k \in(i, j) \\
0, i f k \notin(i, j)
\end{array}\right.
\end{gathered}
$$

$\mathrm{Q}$ is the increases intensity of information, which is constant and greater than $0 . \mathrm{L}_{\mathrm{k}}(\mathrm{t})$ is the length of path that ant $\mathrm{k}$ passes through in this cycle.

In order to speed up the convergence speed of the ant colony algorithm, this paper uses the "incentive suppression strategy" to update the local pheromone. The improvement formula is as follows:

$$
\Delta \tau_{i j}{ }^{\mathrm{k}}(t)=\left\{\begin{array}{ccc}
\frac{Q}{L_{\mathrm{k}}(t)}, & \text { if } & i j \in L_{\mathrm{k}}(t) \\
\varepsilon \frac{Q}{L_{\text {best }}(t)} & \text { if } & i j \in L_{\text {best }}(t) \\
-\varepsilon \frac{Q}{L_{\text {worst }}(t)} & \text { if } & i j \in L_{\text {worst }}(t)
\end{array}\right.
$$

$\Delta \tau_{\mathrm{ij}}^{\mathrm{k}}$ is the information concentration between the node $\mathrm{i}$ and the node $\mathrm{j}$ path in this cycle. $\varepsilon$ is a constant and the $\mathrm{L}_{\mathrm{k}}$ is the path length of the ant $\mathrm{k}$. The $\mathrm{L}_{\text {best }}$ is the length of the optimal path of this cycle and the $\mathrm{L}_{\text {worst }}$ is the length of the worst path of this cycle.

Figure 2 shows the flow chart of improved ACO.

\section{Results and comparisons}

We use MATLAB 2016a to build the simulation platform. The parameters of classical ACO and improved ACO are as follow: the number of maximum iterations $\mathrm{NCmax}=50$, the number of ants $\mathrm{m}=30$, the experimental data set $\alpha=1.1, \beta=9, \rho=0.8$, the initial pheromone $Q=1$, $\mathrm{q} 0=0.4$, the minimum pheromone of evaporation coefficient set $\rho_{\text {min }}=0.3$. The grids in the experimental environmental is a square area by $30 \times 30$. The Figure 3 is the results of global optimal path that was get by using classical ACO and the Figure4 is the results of global optimal path that was get by using improved ACO.

It can be seen from the results that two algorithms can successfully avoid obstacles, but the improved ACO has less inflection points and a shorter path length than the classical ACO.

The results of the convergence curve that were get by using classical and improved ACO are shown in Figure 5 and Figure 6. The average results of two algorithms running 30 times as shown in Table 1.

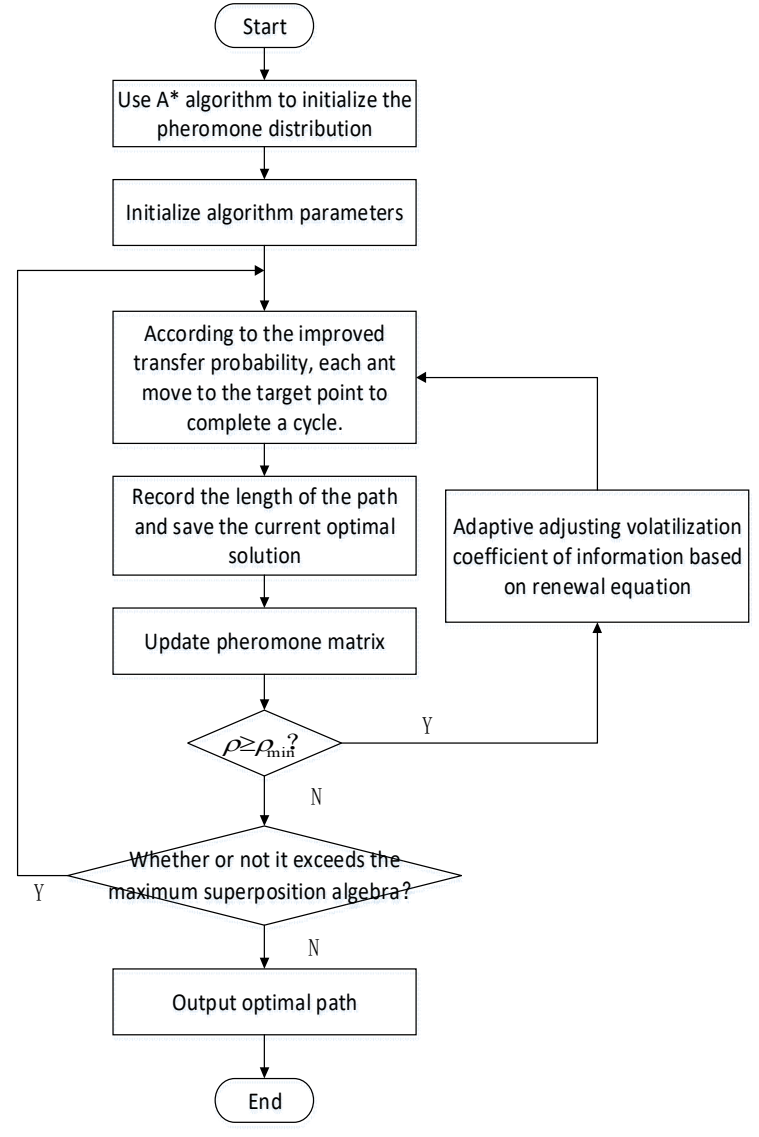

Figure 2. The flow chart of improved algorithm.

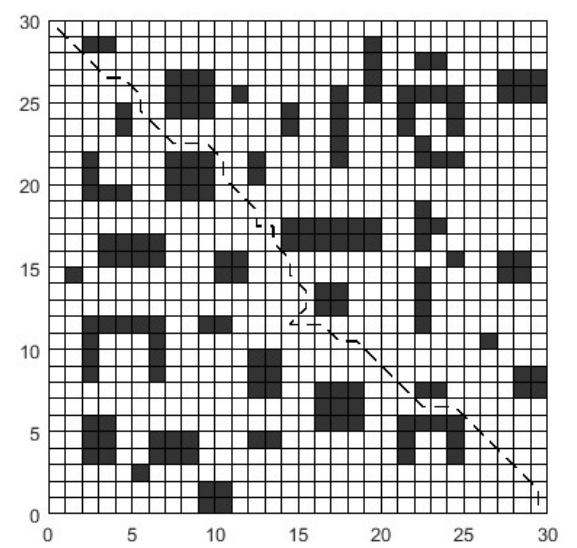

Figure 3. Optimal path of classical ACO.

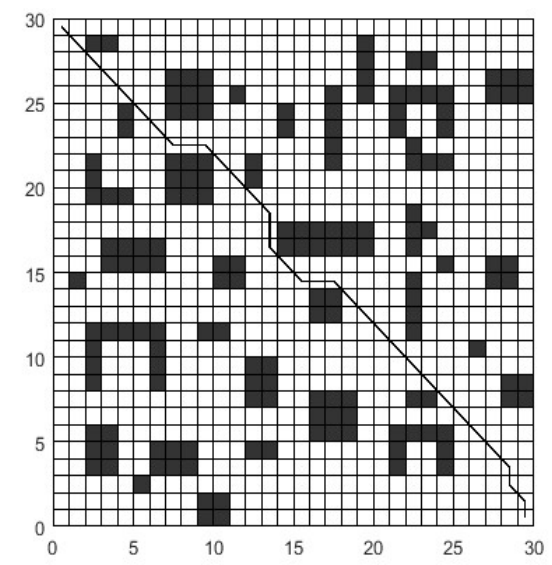

Figure 4. Optimal path of improved ACO. 


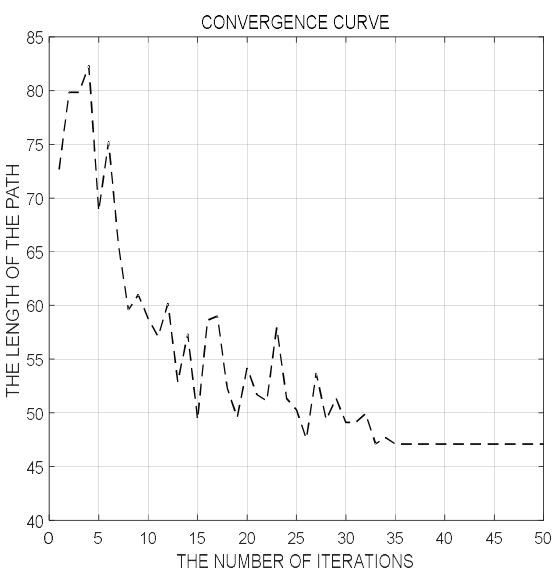

Figure 5. The convergence curves of classical ACO.

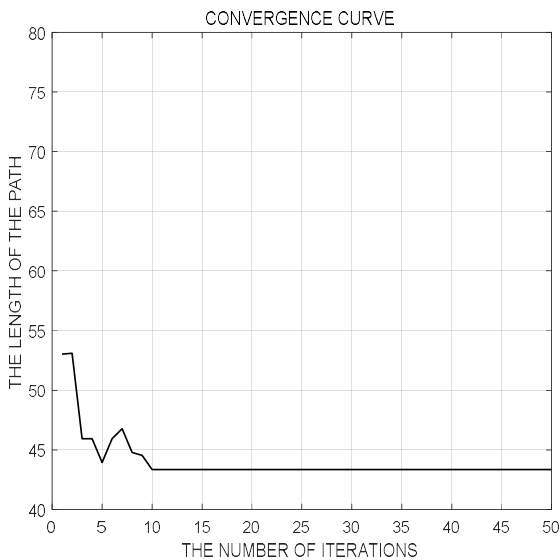

Figure 6. The convergence curves of improved ACO.

Table 1. Comparison of ACO and Improved ACO.

\begin{tabular}{|c|c|c|}
\hline & ACO & $\begin{array}{c}\text { Improved } \\
\text { ACO }\end{array}$ \\
\hline BEST PATH & 47.11 & 43.36 \\
\hline $\begin{array}{c}\text { AVERAGE } \\
\text { PATH }\end{array}$ & 49.88 & 43.59 \\
\hline $\begin{array}{c}\text { THE AVERAGE } \\
\text { NUMBER OF } \\
\text { CONVERGENCE }\end{array}$ & 35 & 11 \\
\hline
\end{tabular}

As can be seen from the experimental results that the classical ACO converges to the optimal path 47.11 with 35 iterations and the improved ACO converges to the optimal path 43.36 with only 11 iterations. Comparing with the classical ACO, the convergent speed of the improved algorithm speed up obviously and the search efficiency improved obviously. with 35 iterations and the improved ACO converges to the optimal path 43.36 with only 11 iterations. Comparing with the classical ACO, the convergent speed of the improved algorithm speed up obviously and the search efficiency improved obviously.

\section{Conclusion}

On the basis $\mathrm{ACO}$ and $\mathrm{A}^{*}$ algorithm, this paper improves the classical ACO from four aspects: the initial pheromone distribution, the selection transfer probability, the pheromone volatilization coefficient and the global pheromone updating rule. The simulation results show that the improved ant colony algorithm can effectively improve the blindness, long search time and local optimality of the classical ant colony algorithm. Therefore, the improved ACO proposed in this paper provides a practical and fast and efficient method for the application of mobile robot planning.

\section{Acknowledgement}

The authors would like to acknowledge the financial support from the projects of National Natural Science Foundation of China (No. 61464001).

\section{References}

1. E Colle, S Galerne. Mobile robot localization by multiangulation using set inversion. ROBOT AUTON SYST. vol. 61, no.1, pp.39-48, 2013.

2. D Lee, J Jeong, HK Yong, BP Jin. An improved artificial potential field method with a new point of attractive force for a mobile robot. International Conference on Robotics and Automation Engineering, pp.63-67, 2017.

3. Z L Ye, M X Yuan, S Cheng. A new explosive immune programming algorithm for mobile robots. Journal of Compute Simulation, vol.30, no.3, pp.323-326, 2013.

4. D X Zhao, Y U Ming, XU Wan. AGV optimal path planning based on genetic algorithms of large fitness value. Computer Engineering and Design, vol.38, no.6, pp.1635-164, 2017.

5. Aamer. Hardware realization of neural network based controller for autonomous robot navigation. IEEE International Conference on Computing Methodologies \& Communication. pp.243-248, 2017.

6. Dorigo M, Gambardella M. A cooperative learning approach to the traveling salesman problem, IEEE Transactions on Evolutionary Computation. vol.1, no.1, pp.5366, 1997.

7. T Tan, S D Liu, Y Zhang. Simulation Research on mobile robot path planning $[\mathrm{J}]$. Journal of Computer Simulation, vol.33, no.8, pp.354-358, 2016.

8. C C Hsu, R Y Hou, W Y Wang. Path Planning for Mobile Robots Based on improved Ant Colony Optimization. IEEE International Conference on Systems, vol.8215, no.2, pp.2777-2782, 2013.

9. H Qu, L W Huang, X Ke. Research on robot path planning based on Improved Ant Colony Algorithm in dynamic environment. Journal of University of Electronic Science and technology of China, vol.44, no.2, pp.260265, 2015.

10. S G Cui , J L Dong, F Liang. The Mixing Algorithm of ACO and GA Based Global Path Planning Method for Mobile Robot . Applied Mechanics\&Materials, vol.494495, pp.1290-129, 2014.

11. H Pan, C Guo, Z Wang. Research for path planning based on improved astart algorithm. International Conference on Information, pp.225-230, 2017.

12. M.Diaz, H.Lombera, E.Martinez, R.Garza. An approach for Assembly Sequence Planning based on Max-Min Ant System. IEEE Latin America Transactions, vol.413, no.4, pp.907-912, 2015. 